in the s-channel: if a given Regge pole $R$ is not coupled to the state $3 \overline{1}$ (or $\overline{4} 2$ )

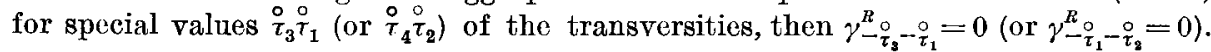

vi) Parity conservation implies that $T_{\{\tau\}}^{s}$ vanishes if $(-1)^{\tau_{1}+\tau_{2}-\tau_{3}-\tau_{4}}=-\eta$, where $\eta$ is the product of the four intrinsic parities. Then the phase

$$
\exp \left[i \frac{\pi}{2}\left(\tau_{1}+\tau_{2}-\tau_{3}-\tau_{4}\right)\right]
$$

in eq. (3) is either $\pm i$ or \pm 1 simultaneously in all nonvanishing amplitudes. In particular, all these amplitudes, in the forward direction, have the same phase, up to a sign, independent of the couplings.

\title{
Photoproduction of Vector Mesons
}

\section{as Virtual Vector Meson-Proton Scattering: Addendum.}

\author{
P. G. O. Freund \\ The Enrico Fermi Institute for Nuclear Studies \\ Department of Physics, The University of Chicago - Ohicago, Ill. \\ (Nuovo Cimento, 48 A, 541 (1967))
}

The Compton scattering cross-section using our result (9) now becomes

$$
\frac{\mathrm{d} \sigma_{\gamma p \rightarrow \gamma_{p}}}{\mathrm{~d} t} \approx\left(X_{\rho}^{2}+X_{\omega}^{2}+X_{\varphi}^{2}\right)^{2} \frac{\mathrm{d} \sigma_{\pi N}}{\mathrm{~d} t} \approx X_{\rho}^{4} \frac{\mathrm{d} \sigma_{\pi N}}{\mathrm{~d} t} \approx 1 \frac{1}{9 \cdot 10^{4}} \mathrm{~d} \frac{\mathrm{d} \sigma_{\pi N^{\nu}}}{\mathrm{d} t}
$$

because $X_{\omega}^{2}$ and $X_{\dot{\varphi}}^{2}$ are $\ll X_{\rho}^{2}$. This is in agreement with our earlier result, P. G. O. Freund: Nuovo Cimento, 44 A, 411 (1966), eq. (3.8) (as it appears in this reference eq. (3.8) contains a misprint: the factor $\left(X_{\rho}^{4}+X_{\omega}^{4}+X_{\psi}^{4}\right)$ should read $\left.\left(X_{\rho}^{2}+X_{\omega}^{2}+X_{\varphi}^{2}\right)^{2}\right)$. For total cross-sections the equivalent of eq. (12) is

$$
\sigma_{\mathrm{tot} \gamma \mathrm{p}} \approx X_{\mathrm{p}}^{2} \sigma_{\mathrm{tot} \pi \mathcal{N}} \approx \frac{1}{300} \sigma_{\mathrm{tot} \pi \mathcal{N}} \approx 70 \mu \mathrm{b}
$$

